\section{Comment on "Thickness and Camber Effects in Slender Wing Theory"}

\author{
Ana Laverón-Simavilla and José Manuel Perales \\ Universidad Politécnica de Madrid, E-28040 Madrid, Spain
}

$\mathrm{N}$ the paper by Plotkin, ${ }^{1}$ first-order corrections to slender wing theory ${ }^{2}$ were developed due to spanwise thickness and camber distributions. The velocity potential $\phi(x, y, z)$ calculated in the paper $^{1}$ actually corresponds to the flow having zero normal velocity at the body contour and a vertical velocity at infinity proportional to the angle of attack $\left[\phi_{z}(x, y,|z| \rightarrow \infty)=U \alpha\right]$. The mentioned velocity potential $\phi$ is not the perturbation velocity potential of the inner flow, $\phi(x, y, z)$, having a vertical velocity $-\alpha U$ at the body contour and zero velocity at infinity. ${ }^{3}$ Therefore, the lift obtained by an integration of the pressure jump over the wing surface. calculated using $\phi$, should have been calculated using $\phi^{\prime}$ in Plotkin's
Eqs. (20-22), the difference in the lift value being

$$
\rho U^{2} S_{z \alpha}
$$

where $S_{b}$ is the area of the final section, which is zero for wings without thickness but should be retained in the case considered in Plotkin's paper.

Particularly, for the elliptical cross-sectional wing solved in Plotkin's paper, ${ }^{1}$ this new term cancels out the perturbation term $\frac{1}{3} \pi A \alpha \varepsilon$ calculated in the paper. which is. finally.

$$
C_{L}=\frac{1}{2} \pi A \alpha
$$

Up to the considered approximation, there is no contribution of the thickness of a wing with the considered shape to the lift.

For the elliptical section wing. Eq. (2) can also be obtained using the Joukowsky conformal transform to map the ellipse into a circle. It is not necessary, for this particular case, to use the expansion in Plotkin's paper ${ }^{1} \mathrm{Eq}$. (4). Therefore, it can be stated that for slender wings with elliptical sections, there is no contribution to the lift due to its thickness.

\section{References}

${ }^{1}$ Plotkin. A., "Thickness and Camber Effects in Slender Wing Theory." AlAA Journal. Vol. 21. No. 12, 1983. pp. 1755-1757.

'Jones, R. T., "Properties of Low-Aspect-Ratio Pointed Wings at Speeds Below and Above the Speed of Sound," NACA Rept. 835. 1946.

${ }^{3}$ Ashley. H., and Landahl. M., Acrodynamics of Wings and Bodies, Addison Wesley. Reading, MA. 1965, Chap. 6.

H. Reed 